\title{
Thoughts and Progress
}

\section{Continuous Delivery of Biomaterials to the Skin-Percutaneous Device Interface Using a Fluid Pump}

\author{
* $\uparrow$ Antonio Peramo, $†$ Cynthia L. Marcelo, \\ $\neq \$$ Steven A. Goldstein, and * $*$ IDavid C. Martin \\ Departments of $*$ Materials Science and Engineering, \\ $\dagger$ Surgery, $\neq$ Biomedical Engineering, and \\ \$Orthopedic Surgery, and IIMacromolecular Science \\ and Engineering Center, University of Michigan, \\ Ann Arbor, MI, USA
}

\begin{abstract}
We have developed an in vitro culture system composed of organotypic human skin explants interfaced with titanium rods attached to a fluid pump. This device was designed to mimic the process of natural mucosa delivery at the point where a rigid, permanent object penetrates living skin. Full thickness human breast skin explants discarded from surgeries were cultured at different time points at the air-liquid interface. The skin specimens were punctured to fit at the bottom of hollow cylindrical titanium rods. Sodium lauryl sulfate (SLS) was delivered continuously to the specimens through the rods by using an attached fluid pump. Histological analysis of the skin explants as well as no-pump controls was then performed. Our results show substantial differences between controls, where no material was pumped at the interface of rod-skin, and specimens treated with SLS, indicating that the technique of pumping the material is effective in producing observable epithelial changes. These results suggest that an adaptation of this type of device may be useful for the treatment of complications arising from the contact between tissues and percutaneous devices in vivo. Key Words: Medical device-Skin biomaterial-Implant interface-Osseointegration-Implants.
\end{abstract}

The use of percutaneous medical devices passing for extended periods of time across the skin has increased substantially in the last few years. In conjunction with the extended use of catheters and a general tendency toward minimally invasive medical procedures, the need for a stable interface between

doi:10.1111/j.1525-1594.2009.00931.x

Received March 2009; revised July 2009.

Address correspondence and reprint requests to Dr. Antonio Peramo, Department of Surgery and Department of Materials Science and Engineering, University of Michigan, Ann Arbor, MI 48109, USA. E-mail: aperamo@umich.edu internal tissues and percutaneous devices has become of higher importance. There is also a need to develop rapid and reproducible testing methods to evaluate the interfaces between these devices and internal tissues and organs because although animal models are available to evaluate some of these percutaneous devices (1), it is difficult to perform clinical studies (2). Percutaneous devices suffer from failures due to breakdown or infections of the skin at the implant-soft tissue interface. These problems prevent the formation of a stable dermal and epidermal seal around the implants $(3,4)$. Examples of such devices that are either already in clinical use or are being actively developed include limb prostheses or glucose sensors $(5,6)$. In all of these devices, the risk of long-term inflammation, infection, and other reactive tissue responses provides a serious limit to their functionality (7). The inflammation and infection can spread from the dermal and epidermal layers to the interior of the body, particularly to the bone (8).

To start addressing these issues with percutaneous devices, we have previously presented a system that permits the in vitro analysis of the interface between skin and external devices (9). The system was used to observe changes in tissue morphology of the skin in contact with pins in the presence or absence of a variety of materials in solution. In this previous work, the material was delivered discontinuously at the skin-device interface so we questioned whether a continuous delivery of the solutions would be better than the discontinuous delivery. This process might help improve the long-term performance of percutaneous devices.

We present here the in vitro culture system shown in Fig. 1 that allows the delivery of a material to the skin-device interface by a continuously controlled pumping. The use of this interface provides a model to evaluate strategies for creating a stable, long-term connection with living skin and chronic percutaneous devices. Our hypothesis is that the delivery of specific biomaterials at this interface will create a dynamic, slowly flowing matrix for skin biointegration and the local administration of drugs or antimicrobials. Our essential concept is to incorporate a reservoir of material that can be slowly extruded at the implantskin interface to protect the underlying tissue layers from the possibility of infection and inflammation. To 


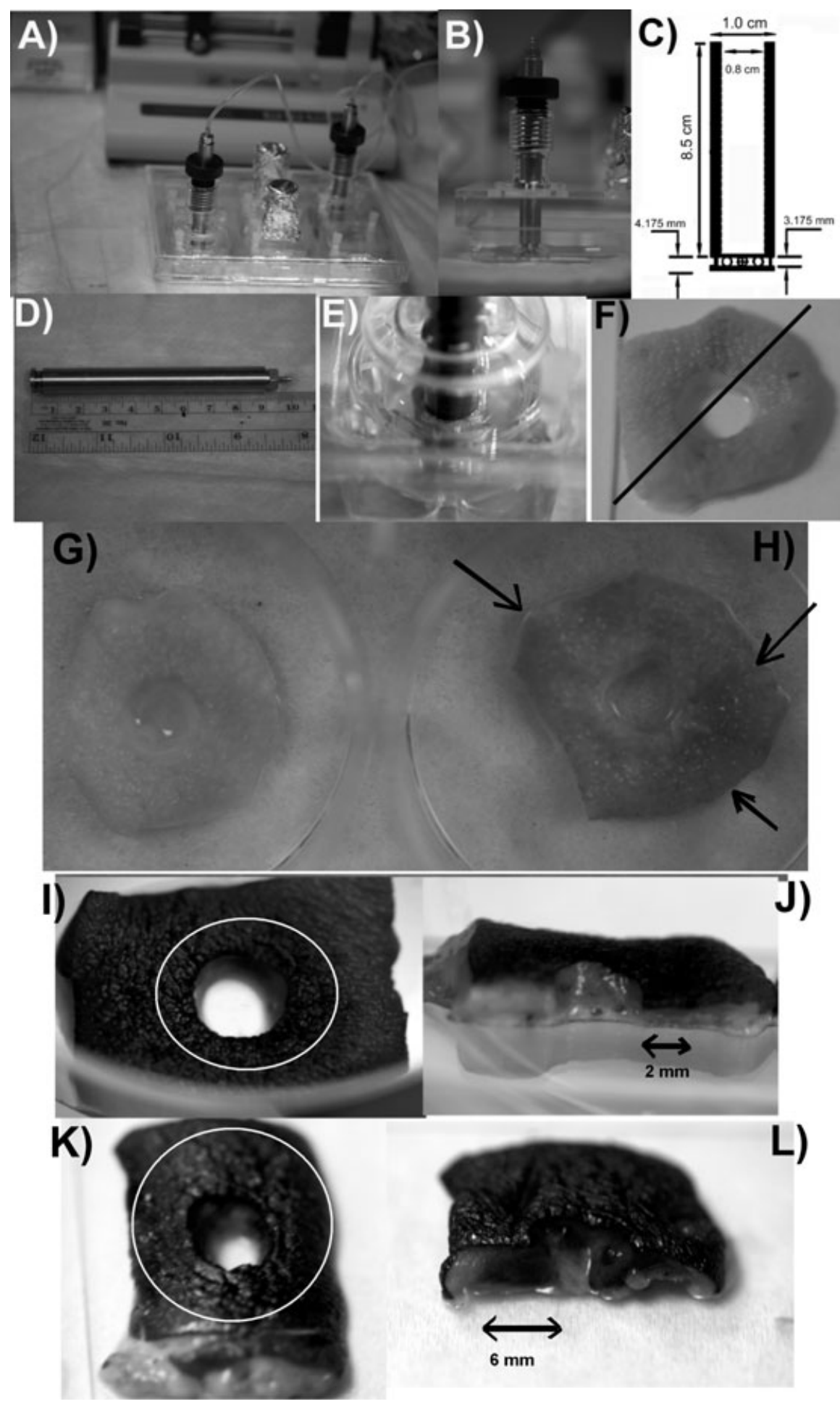

FIG. 1. The top part of the figure shows the schematic of the culture system used to pump materials to the interface of titanium rods and human skin explants using an air-liquid interface culture and an in-house designed lid to accommodate the titanium rods. Fig. $1 \mathrm{~A}$ is an overall view of the system, with culture plate, lid, rods, tubing, and pump. Fig. 1B,D are close-ups of the rods and 1C shows their dimensions. Fig. 1E is a close-up of a specimen inserted in the rod. Fig. 1F indicates how the specimens were cut for histology. The bottom of this figure shows optical photographs of skin specimens. In Fig. 1G, a control specimen ROD cultured for 3 days is shown. In Fig. $1 \mathrm{H}$, an SLS specimen cultured for 5 days is shown. The arrows in Fig. $1 \mathrm{H}$ indicate extensive areas of visible tissue deterioration. The rest of the pictures show specimens where a solution of methylene blue was pumped continuously for 1 day (Fig. $11, \mathrm{~J}$ ) or 5 days (Fig. $1 \mathrm{~K}, \mathrm{~L}$ ) at a rate of $0.1 \mu \mathrm{L} / \mathrm{min}$. The extent of methylene blue diffusion is indicated by the circles. In the dermis, the extent of methylene blue is indicated by the arrows. The diameter of all perforations is $8 \mathrm{~mm}$. 
our knowledge, this concept of a continuously replenished fluid interface that mimics the regenerative nature of living tissue is unprecedented, and may have other important implications for a variety of biomedical devices.

\section{MATERIALS AND METHODS}

\section{Skin preparation and culture system and pumping}

Human skin was obtained and prepared for the experiments as we previously reported elsewhere (9). All skin specimens were punctured with an $8-\mathrm{mm}$ diameter sterile biopsy punch (except for specific controls, described next). Experiments consisted of a culture plate containing up to four different skin specimens, which varied depending on the experiment: plates with skin specimens with a rod but no pumping (ROD) and plates with skin specimens with rod and sodium lauryl sulfate (SLS) pumped. In addition, a primary, noncultured control specimen at 0 days (CNTRL-0D) was selected from the original skin. After cleaning, this CNTRL-0D was immediately prepared for tissue analysis, as described next. Figure 1 shows the experimental chamber with its components. The glass lids that covered the culture plates were designed and produced in-house. The body of the lid itself is solid, created from a noncorrosive material such as glass that permits visualization of the biological tissue underneath. The chamber used had four apertures for four cylindrical rods that formed air-tight seals to prevent contaminants entering the reservoirs. Medium changes on wells were performed using the small feeding opening on the lid located next to each cylinder aperture, which were otherwise permanently closed. All materials used were autoclaved before use in each experiment. Titanium rods, photographed in Fig. 1B,D (1.0 cm OD and $10 \mathrm{~cm}$ long, McMaster-Carr, Atlanta, GA, USA) were hollow, with $0.8 \mathrm{~cm}$ ID. These hollow rods had four orifices machined at the bottom of the rod for delivery of the biomaterial. The dimensions of these rods are shown in Fig. 1C. For cleaning and elimination of particulates or extraneous materials, the rods were sonicated for $30 \mathrm{~min}$ with Liquinox and Citranox (VWR, Westchester, PA, USA) and autoclaved before use on each experiment. A $1 \%$ solution of the surfactant SLS (Sigma-Aldrich, St. Louis, MO, USA) was pumped to specimens termed SLS while ROD specimens did not receive any material. The solution was pumped continuously during the duration of the experiments $(1,3$, or 5 days) at a rate of $0.1 \mu \mathrm{L} / \mathrm{min}$, stopping only briefly to allow medium changes in the cultures. At this rate, the total volume delivered per day was $144 \mu \mathrm{L}$. Two additional pumping rates $(0.01$ and $1 \mu \mathrm{L} / \mathrm{min}$ ) were additionally tested to best determine the correct pumping rate for the rest of the experiments. The tubing used was Tygon R-3603 (VWR) and the pump was a dual syringe microdialysis pump (SP101i, World Precision Instruments, Sarasota, FL, USA). The syringes used were from Hamilton Company (Reno, NV, USA). Given the small volumes to be pumped during the 5-day period, the tubing and the rods were first filled with the materials before the start of the experiment. To visually inspect the approximate distribution of the pumped materials, methylene blue (MW 320) was pumped for up to 5 days at a rate of $0.1 \mu \mathrm{L} / \mathrm{min}$.

\section{Analysis of skin specimens}

Histological evaluation of collected specimens was performed with hematoxylin and eosin (H\&E) and with Masson's Trichrome. For all specimens containing a perforation, tissue sections were cut from the injured areas as depicted in Fig. 1F, through the center of the specimens. Blind qualitative histological analysis was performed by two independent investigators. For histological analysis, biopsies were fixed in $4 \%$ phosphate buffered paraformaldehyde for $24 \mathrm{~h}$, routinely dehydrated and paraffin embedded. Serial sections were obtained at $5 \mu \mathrm{m}$. In addition, immunohistochemical analysis of epidermal cell differentiation (involucrin) was performed. For keratinocyte differentiation, slides were first treated for $5 \mathrm{~min}$ with proteinase $\mathrm{K}$ to induce antigen retrieval, and involucrin was detected by light microscopy with a mouse horseradish peroxidase conjugated monoclonal antibody against involucrin (1:100, 30 min, Leika Microsystems, Deerfield, IL, USA) and counterstained with hematoxylin. For light microscopy analysis, images were taken using a Nikon E800 microscope (Melville, NY, USA).

\section{RESULTS}

Figure 1 shows details of the experimental setup and an average representation of the skin specimens (Fig. 1G,H) and the extent of methylene blue distribution (Fig. 1I-L) at different time points. Figure 1A presents an overall view of the system, with culture plate and lid and two rods with tubing connected to the pump, in the background. In this specific lid design, up to four rods can be simultaneously used. Figure 1B,D are close-ups of the rods and $1 \mathrm{C}$ shows their dimensions. Figure 1E is a close-up of a specimen inserted in the rod. For histology, Fig. 1F shows that the specimens were cut tracing a line across the center of the perforation. At 3 days, ROD specimens (Fig. 1G) appeared viable and in good general 


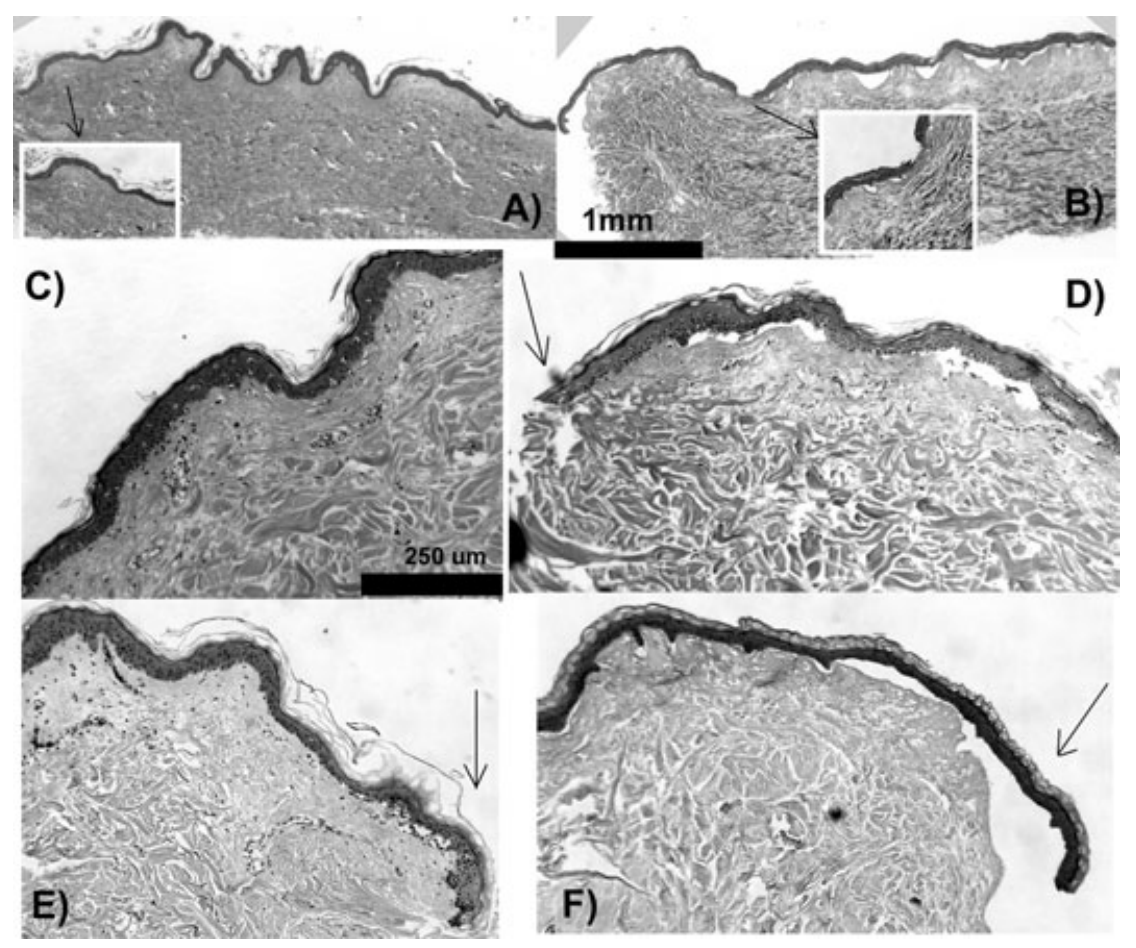

FIG. 2. (A) and (B) are low magnification images of Masson's trichrome stained specimens, while the rest are hematoxylinand eosin-stained high magnification pictures of skin specimens cultured using our system. Arrows indicate the location where the rods were placed except for specimen (C), cultured without a rod or perforation. (A) shows a 3-day ROD control specimen while (B) shows a 3-day cultured specimen with sodium lauryl sulfate (SLS) with selected areas shown in the insets. (D) is a 5-day control, uncultured specimen (cultured without rod), (E) is a 5-day ROD-cultured specimen, $(F)$ is a 3-day ROD-cultured specimen, and $(F)$ is a 3-day SLS-cultured specimen. condition but SLS specimens (Fig. 1H) appeared substantially deteriorated. While denaturation was evident for SLS specimens, this was not apparent for ROD specimens. There was clear indication of the destruction of the whole SLS specimen at great distances from the rim, evident by a general darkening of the epidermal layers, in extensive areas indicated by arrows. These specimens appeared to be dissolving away and looked spongiform. The bottom part of Fig. 1 shows the changes in the intensity of the methylene blue dye after being pumped for 1 (Fig. 1I,J) or 5 days (Fig. $1 \mathrm{~K}, \mathrm{~L}$ ). The areas within the rings show the approximate extent of the distribution of the dye, extending further away from the rim when pumped for longer periods. At the dermis level, this is also demonstrated by comparing Fig. 1J,L. The extension of the distribution corresponds approximately with the areas visible in the stratum corneum, albeit it appears that when pumped for longer periods the dye intensity increased proportionally closer to the rim. The linear extent of the distribution of the dye was approximately $2 \mathrm{~mm}$ from the rim at day 1 and around $6-8 \mathrm{~mm}$ at day 5 .

Figure 2 shows a panel of images containing high and low magnification histology images of representative ROD- and SLS-treated specimens. Figure 2A shows a low magnification picture of a Masson's trichrome stained ROD specimen, that can be compared with an SLS-treated specimen in Fig. 2B. The insets show some details at higher magnification. The overall effect of SLS is easily observable all along the tissue, both at the epidermal and dermal compartments.

Figure 2C,D are presented to show the effect of the percutaneous implant versus a control with no implant. Figure $2 \mathrm{C}$ is an $\mathrm{H} \& \mathrm{E}$ section of a specimen cultured for 5 days without a titanium rod, while Fig. 2D shows a ROD specimen. While the nonperforated specimen shows some signs of deterioration, particularly detectable with the presence of vacuoles and piknotic cells, the overall epidermal and dermal structures are still well preserved. In contrast, the 5-day cultured ROD specimen has more piknotic cells, shows separation of the dermal-epidermal junction close to the rod, and the dermis presents widening gaps. Figure 2E,F are presented to compare the effect of SLS versus a ROD control, in a 3-day period. The areas in close contact with the rods are indicated with arrows. In the ROD control, the presence of the rod leads to some separation of the epidermis and presence of piknotic cells, but except in very close areas (up to approximately $250 \mu \mathrm{m}$ ), the tissue remains fairly normal. This is totally in contrast to the SLS-treated specimens that are severely damaged in all areas. It should be noted that the SLS-treated specimen does not show piknotic cells on any areas. The stratum corneum is swollen, while the dermis appears contracted. 


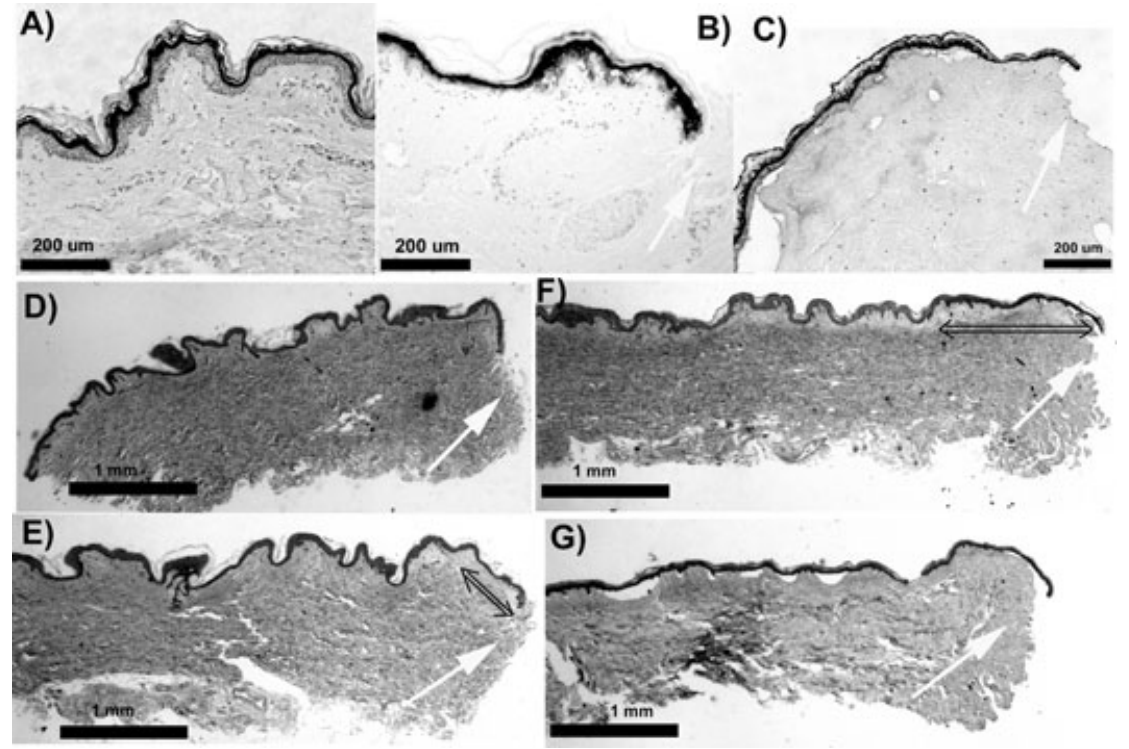

FIG. 3. Effect of sodium lauryl sulfate (SLS) on cellular differentiation (A-C) and hematoxylin and eosin histological analysis of effect of SLS at two different timepoints $(D-G)$. The white arrows in $B-G$ indicate the location where the rods were placed. The presence of the differentiation marker involucrin is shown on a CNTRL-OD specimen (A), a 3-day RODcultured specimen (B), and a 3-day SLS specimen (C). In the bottom part of this Figure, the left column shows ROD control specimens cultured for 1 day (D) or 3 days $(E)$, while in the right column SLS specimens cultured for $1(F)$ or 3 days $(G)$ are shown. The double-headed black arrows in images (E) and (F) indicate the approximate distances from the edge of the tissue where epidermal changes are more visible. For the control specimen (D), the changes appear negligible while for SLStreated specimen $(\mathrm{G})$, the changes extend to the whole section.
Figure 3 presents a composite panel of images showing cellular differentiation of the skin in contact with the rods, treated or not with SLS (top part) and the effect of pumped SLS at two different time points and a comparison with ROD specimens (bottom part). The presence of the differentiation marker involucrin (Fig. 3A) is shown for a CNTRL-0D control. Involucrin functions as intermolecular crossbridge of the keratinocyte-cornified envelope and is normally found in the upper spinous layers (10). When the keratinocytes are hyperproliferative, involucrin would be present in several (if not all) epithelial layers except for the basal layer, given that the epidermis is rapidly differentiating. Involucrin staining in the CNTRL-0D control can be compared with a 3-day ROD specimen (Fig. 3B) and with a 3-day SLS specimen (Fig. 3C). The arrows indicate the position of the rods. The thinning of the epidermis observed in SLS specimens makes difficult a complete comparison. For ROD specimens, involucrin is observed in the lower layers of the epidermis. Figure 3D-G shows the effect of pumping SLS at two different time points. The white arrows in the images indicate the presence of the rods while the doubleheaded black arrows indicate the approximate distance at which the tissue appears to "sense" the presence either of the rod alone or the rod plus the SLS delivered. While for a ROD at 1 day the effect of the rod is barely observable, changes are easily observable up to $250 \mu \mathrm{m}$ when cultured for 3 days. In contrast, when the SLS solution is pumped for 1 day, the combined effect of the presence of the rod and the SLS is seen in the tissue beyond the $1 \mathrm{~mm}$ mark for 1 day, and several millimeters when the specimens are cultured for 3 days.

\section{DISCUSSION}

We have obtained results from an in vitro prototype of a device whose final purpose is to provide a method for the continuous delivery of materials at the skin-percutaneous device interface. As constructed in this prototype, the material is stored in a reservoir that will require occasional replenishment. Our intention is to engineer a strategy that will help percutaneously implanted devices to be in contact with skin layers for very long periods of time, minimizing the immunological response from the host and biofilm formation on the device, inhibiting scar formation at the interface, preventing infection, and serving as conduit for drug delivery to the surrounding tissue.

The concept of slowly pumping a material at the skin-external device interface derives from the idea of replicating the observed release of biomaterials in epithelial tissues and junctions in the body, which slowly release mucins and other solutions to help prevent infection (11). In designing this novel system, several parameters were taken into account to be as close as possible to in vivo conditions. In addition to using human skin explants, the material of the rods was titanium and the dimensions of the rods are similar to the ones that could be implanted in limbs in humans as percutaneous devices (12). The materials pumped (SLS and methylene blue) were continuously delivered to the specimens 
through the rods by using an attached fluid pump, as demonstrated in Fig. 1. After culturing, histological analysis of the skin explants was performed. The rods used were manufactured with a small groove at the bottom where the holes for delivery were located. The groove allows the skin to be put in place more tightly but the skin did not clog the holes of the rods permanently, allowing the pumped fluid to be continuously extruded. To maximize the interaction of the extruded fluid with the tissue, the rods were designed to allow for the delivery to take place at the level of the dermis.

With no previous information regarding the pumping rates, we first wanted to ensure that we could observe the pumped materials reaching the surface of the stratum corneum over the time of the experiment. This will be considered the higher end of a possible range of pumping rates and was designed to be able to obtain accelerated results given the nature of the in vitro experiments. An average measured thickness of $4 \mathrm{~mm}$ was in the upper limit of the skin thickness used for the calculation of the pumping rates. Three fluid rates were preliminarily tested: $1 \mu \mathrm{L} / \mathrm{min}, 0.1 \mu \mathrm{L} / \mathrm{min}$, and $0.01 \mu \mathrm{L} / \mathrm{min}$. In this later case, the total volume extruded is $72 \mu \mathrm{L}$, thus there was no guarantee that the fluid would reach the top of the skin, depending on specimen thickness and this pumping rate was not used. Fluid rates of $1 \mu \mathrm{L} /$ min provided a volume over the 24 -h period that was considered inappropriate for the experimental setup, given the small dimensions of the wells where the skin is cultured and were also not used. Thus, for the experiments, a medium fluid rate of $0.1 \mu \mathrm{L} / \mathrm{min}$ was finally used to ensure that the entire distance (from dermis to stratum corneum) would be covered over the 5-day period with a total volume discharged of $720 \mu \mathrm{L}$.

Assuring the delivery was the central requirement imposed to the system, rather than emulation of a possible biologically relevant pumping rate. In this sense, a distinct and attractive possibility would have been to try to emulate epidermal growth by pumping the fluid at the same average rate at which the cells of the basal layer end up being shed in the stratum corneum. The only part of the skin that grows is the epidermis, which has a thickness in the range of 75-125 microns. It is assumed that this process takes up to 45 days. A rough calculation indicates that this represents a linear displacement of a 0.002 microns/ $\mathrm{min}$. This would be equivalent, using our system, to a fluid rate of $0.00015 \mu \mathrm{L} / \mathrm{min}$ and a total volume pumped over a period of 5 days of $\sim 1 \mu \mathrm{L}$. It is not clear whether precisely replicating epidermal growth rate would be a good objective, but it appears experi- mentally it would not be easy to match, unless the size of the injury and the whole device were drastically reduced, which is not realistic given that the purpose of our experiments was to perform in vitro tests using injuries and percutaneous devices of realistic dimensions.

To provide visual observation of the extent of material diffusion in the dermis and epidermis, experiments using a highly intense dye of low molecular weight were performed. Methylene blue was pumped at a rate of $0.1 \mu \mathrm{L} / \mathrm{min}$ for 1 or 5 days and the results are shown in Fig. 1. As expected, longer pumping periods allowed for an extended distribution of the material away from the rim of the perforation. It must be noted, however, that this distribution is expected to be material-dependent and should be analyzed for each specific product to be delivered. The effectiveness of SLS as a test material for our experiments is evident in the pictures of Fig. $1 \mathrm{G}-\mathrm{L}$, where the surfactant effect of the product is visually observable at 3 days all over the specimen. SLS is a well-known surfactant, which has been extensively used in experimentation with skin in vitro (13). Our continuous pumping delivering the product from the dermis level appeared to exert a rapid disruption of the dermal-epidermal junction. This is observed at different distances and time points (Figs. 2B,F and 3F,G).

We also wanted to briefly analyze how the tissue could sense the presence of the percutaneous device and the pumped material over time. This is shown in Fig. 3D-G. At 1 day, the skin does not seem to sense or be affected by the presence of the rod (Fig. 3D), thus the changes observed in Fig. $3 \mathrm{~F}$ are due only to SLS. This damage extends far away from the rim at 3 days. The effect of the rod alone is also cumulative over time, as is observed by comparing Figs. 3D,E and 2D. A close analysis of these specimens indicates that the effect of the rod is to deteriorate the skin at increased linear distances from the rim over time, but the intensity of the deterioration (which qualitatively can be described as appearance of piknotic cells) appears to be distributed equally all along the tissue. In other words, the tissue does not appear more deteriorated closer to the rim than at greater distances, when the specimens are cultured with the rods over increased periods of time.

In addition to the regular H\&E histological characterization, we performed a basic analysis of cellular differentiation with the differentiation marker involucrin in the specimens, but did not attempt to be exhaustive in this analysis. As seen in our experiments, involucrin is found directly above the basal cell layer through the stratum corneum in 
normal CNTRL-0D samples (Fig. 3A), indicating that the keratinocytes within the epidermis are differentiating. After 3 days in culture with the rods (Fig. 3B), the involucrin distribution is different than in the normal CNTRL-0D specimens. This pattern of involucrin staining has also been reported in skin substitutes grafted to athymic mice (14). This increased presence of involucrin is also observed when SLS is pumped continuously for 3 days (and has been seen in vivo at 3 days [15]), although it was experimentally more difficult to observe in our skin sections due to the thinning of the epidermis and the disruption of the dermal-epidermal junction.

\section{CONCLUSIONS}

In summary, we have shown that the skin specimens interfaced with titanium rods in vitro react significantly differently when they are cultured in the presence of a pumped material, in this case SLS. We emphasize that this system can be adapted and tested with any material used in the fabrication of percutaneous devices and that any appropriate material, including drugs, can be pumped. This type of treatment may be applicable to tissue areas in contact with percutaneous devices in vivo, with the expectation that the delivery of the materials in a manner similar to the natural extrusion performed by tissue mucosas may help in better skin integration with the percutaneous devices, and consequently help in preventing infection and in the regeneration of the tissues around the implants.

Acknowledgments: We thank Harald Eberhart, College of Engineering, University of Michigan, for help with the glass lid design. We also thank Chris Strayhorn, Dental School Histology Core Facility, University of Michigan, for help in immunostaining techniques. This report is presented as part of the research efforts within the Army Research Office Multidisciplinary University Research Initiative award on Bio-Integrating Structural and Neural Prosthetic Materials and we gratefully acknowledge the funding provided. Invention disclosures related to these percutaneous delivery devices have been filed with the University of Michigan Technology Transfer Office and provisional patents have been filed with the U. S. Patent and Trademark Office.

\section{REFERENCES}

1. Moroni A, Orienti L, Stea S, et al. Improvement of the pinbone interface with hydroxyapatite coating. An in vivo longterm experimental study. J Orthop Trauma 1996;10:236-42.

2. Masse A, Bruno A, Bosetti M, Biasibetti A, Cannas M, Gallinaro P. Prevention of pin track infection in external fixation with silver coated pins: clinical and microbiological results. J Biomed Mater Res B Appl Biomater 2000;53:600-4.

3. Winter GD. Transcutaneous implants: reactions of the skinimplant interface. J Biomed Mater Res 1974;8:99-113.

4. Von Recum AF. Applications and failure modes of percutaneous devices: a review. J Biomed Mater Res 1984;18:323-36.

5. Pickup JC, Hussain F, Evans ND, Sachedina N. In vivo glucose monitoring: the clinical reality and the promise. Biosens Bioelectron 2005;20:1897-902.

6. Meulenbelt HE, Dijkstra PU, Jonkman MF, Geertzen JH. Skin problems in lower limb amputees: a systematic review. Disabil Rehabil 2006;28:603-8.

7. Luttikhuizen DT, Harmsen MC, Van Luyn MJ. Cellular and molecular dynamics in the foreign body reaction. Tissue Eng 2006;12:1955-70.

8. Aro HT, Markel MD, Chao EYS. Cortical bone reaction at the interface of external fixation half-pins under different looking conditions. J Trauma 1993;35:776-85.

9. Peramo A, Marcelo CL, Goldstein SA, Martin DC. Novel organotypic cultures of human skin explants with an implanttissue biomaterial interface. Ann Biomed Eng 2009;37:401-9.

10. Bedoni M, Sforza C, Dolci C, Donetti E. Proliferation and differentiation biomarkers in normal human breast skin organotypic cultures. J Dermatol Sci 2007;46:139-42.

11. Meyer-Hoffert U, Hornef MW, Henriques-Normark B, et al. Secreted enteric antimicrobial activity localizes to the mucus surface layer. Gut 2008;57:764-71.

12. Branemark PI, Albrektsson T. Titanium implants permanently penetrating human skin. Scand J Plast Reconstr Surg 1982; 16:17-21.

13. Varani J, Fligiel SE, Perone P, Inman DR, Voorhees JJ. Effects of sodium lauryl sulfate on human skin in organ culture: comparison with all-trans-retinoic acid and epidermal growth factor. Dermatology 1993;187:19-25.

14. Medalie DA, Eming SA, Tompkins RG, Yarmush ML, Krueger GG, Morgan JR. Evaluation of human skin reconstituted from composite grafts of cultured keratinocytes and human acellular dermis transplanted to athymic mice. J Invest Dermatol 1996;107:121-7.

15. Törmä H, Lindberg M, Berne B. Skin barrier disruption by sodium lauryl sulfate-exposure alters the expressions of involucrin, transglutaminase 1 , profilaggrin, and kallikreins during the repair phase in human skin in vivo. $J$ Invest Dermatol 2008;128:1212-9. 